\title{
Phonon and Electronic Non-radiative Decay of Excitons in Carbon Nanotubes
}

\author{
Vasili Perebeinos and Phaedon Avouris \\ IBM Research Division, T. J. Watson Research Center, \\ Yorktown Heights, New York 10598 \\ (Dated: November 6, 2018)
}

\begin{abstract}
We investigate theoretically the rates of non-radiative decay of excited semiconducting nanotubes by a variety of decay mechanisms and compare with experimental findings. We find that the multiphonon decay (MPD) of free excitons is too slow to be responsible for the experimentally observed lifetimes. However, MPD lifetimes of localized excitons could be 2-3 orders of magnitude shorter. We also propose a new decay mechanism that relies on a finite doping of nanotubes and involves exciton decay into an optical phonon and an intraband electron-hole pair. The resulting lifetime is in the range of 5 to $100 \mathrm{ps,} \mathrm{even} \mathrm{for} \mathrm{a} \mathrm{moderate} \mathrm{doping} \mathrm{level.}$
\end{abstract}

Semiconducting single-walled carbon nanotubes (CNTs) are one-dimensional, direct band-gap materials with strongly-bound exciton states, that are actively being explored for technological applications ${ }^{1}$. The potential usefulness of CNTs as an optical material depends, to a large extent, on their luminescence efficiency, which is determined by their radiative and nonradiative lifetimes. The CNT radiative lifetime has been calculated ${ }^{2,3}$ to be in the range of $1-10 \mathrm{~ns}$. The measured lifetime, however, is much shorter, in the range of 10 to $100 \mathrm{ps}^{4}, 5,6,7,8,9$, indicating the presence of an efficient non-radiative decay channel(s). The nature of the dominant non-radiative decay path has been the subject of debate $\frac{10,11,12}{}$. Low energy, 'dark' exciton states $2,3,13,14$ have been proposed as the cause for the small yield, but the small dark-bright exciton splitting does not support this conclusion ${ }^{12}$. It is also known that excitons in bulk semiconductors can be localized at structural defects, impurities, grain boundaries and other heterogeneities ${ }^{15}$. Analogous exciton localization can occur in CNTs. In fact, the quasi-1D, single atomic layer structure of single-walled CNTs makes them quite sensitive to potential fluctuations in their environment, e.g. trapped charges on an insulating substrate, adsorbed species, etc. Recent photovoltaic studies of CNTs have directly revealed such potential fluctuations ${ }^{16}$. Even in solution, the coating of surfactant-coated CNTs may not be homogeneous, leading to potential fluctuations, and in otherwise perfect tubes their ends can act as exciton traps. Photoluminescence studies of surfactant-coated CNTs have indeed provided strong evidence for the formation of localized excitons $17,18,19$. The strong effect of the environment on the non-radiative decay is most clearly shown by the fact that no fluorescence was detected from CNTs on an $\mathrm{SiO}_{2}$ substrate ${ }^{20}$, but the tubes luminescence strongly when suspended 21 . It is also known that, although not intentionally doped, CNTs on most substrates in air behave as p-type semiconductors ${ }^{11}$. Suspended CNTs, on the other hand, usually show intrinsic behavior ${ }^{22}$. Furthermore, CNTs on insulators show pronounced hysteretic effects due to shifts of their Fermi level (field doping) by trapped charges $23,24,25$. Analogous charge-transfer doping effects have been suggested for a variety of adsorbed gases on $\mathrm{CNTs}^{26}$.

It is clear that a variety of factors and environmental influences can affect the non-radiative decay of excited nanotubes. In an effort to evaluate their contributions, we present in this Letter a theoretical study of the efficiency of non-radiative decay pathways of nanotubes involving purely multi-phonon decay as well as electronic decay mechanisms. We examine the decay behavior of free-exciton nanotube states, trapped (localized) excitons, and excitons in doped nanotubes, i.e. nanotubes whose Fermi level does not coincide with the neutrality level. In the latter case, we propose and evaluate a novel, very efficient, phonon-assisted indirect exciton ionization (PAIEI) mechanism. Our calculations indicate that the combination of localized exciton MPD and the PAIEI mechanism allow us to explain the range of available experimental data on the non-radiative lifetime. Furthermore, the calculations suggest that, in principle, a high emission quantum yield can be achieved in intrinsic, small diameter tubes.

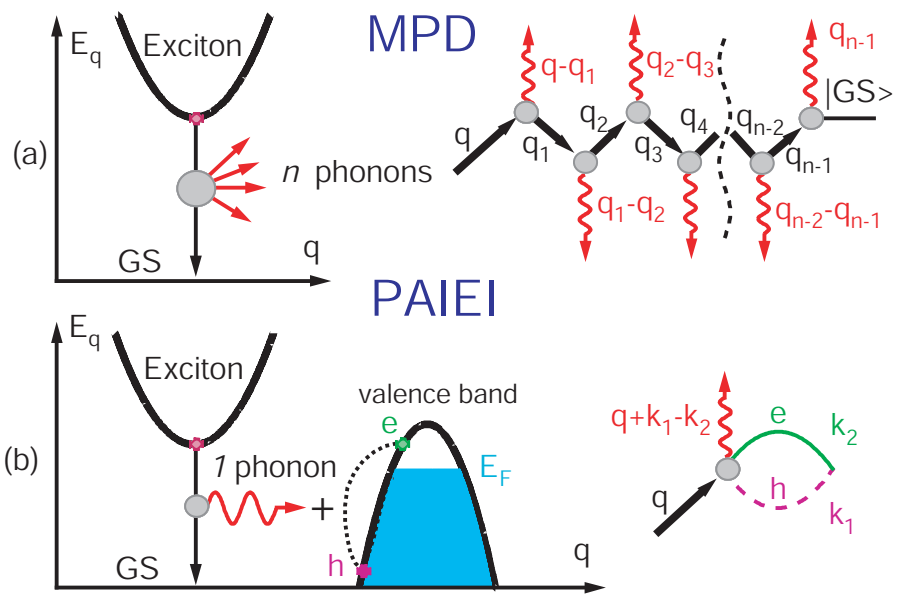

FIG. 1: Schematics of the exciton decay mechanisms: (a) Multiphonon Decay (MPD) and (b) Phonon-Assisted Indirect Exciton Ionization (PAIEI) in p-doped CNTs.

First, we explore the MPD decay process for free excitons schematically shown in Fig. 17. The MPD decay of 
excited CNTs to the ground state has been proposed 10 , as being plausible for CNTs because of the high energy C-C stretching phonon (G-mode, $\hbar \omega \approx 0.2 \mathrm{eV}$ ) and its strong electron-phonon coupling 27,28 .

To calculate the MPD rate we need to evaluate the $n$th order diagram depicted in Fig. 1 ${ }^{29}$. The electronphonon Hamiltonian involves both intraband and interband $\left(M^{4}\right)$ electron-phonon couplings. It is obtained using the Su-Schreiffer-Heeger model as in ref. .27 including a bond-bending coupling term 29,30 . The exciton-phonon coupling is derived from the electron-phonon coupling using the two-particle exciton wavefunctions calculated from the Bethe-Salpeter Equation as in ref $\underset{13,29}{2}$.

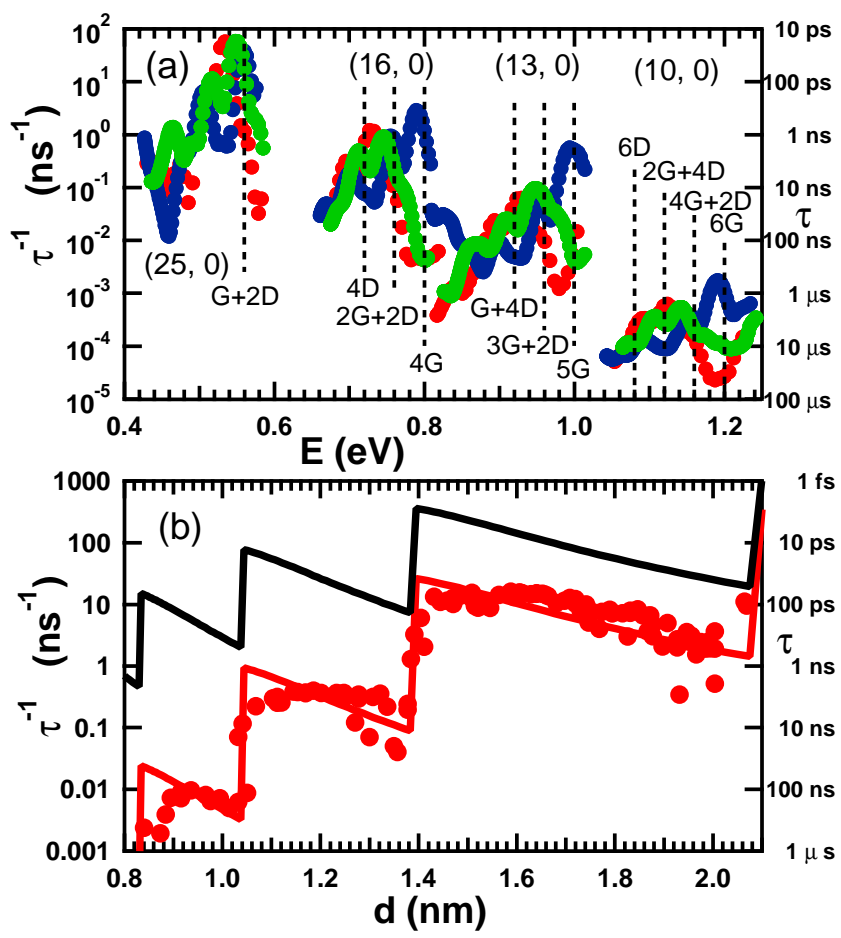

FIG. 2: (a) MPD rate in four nanotubes as a function of exciton energy calculated with $\varepsilon=3.3$ for the four lowest energy bands: bright (red), dark (blue), doubly degenerate dark (green). The vertical dashed lines show characteristic energies of different combinations of the zone-center $\mathrm{G}$ mode and even numbers of the zone-boundary D modes. (b) averaged MPD rate in 118 tubes as a function of tube diameter (red circles). The solid red curve is a fit to Eq. (10) reduced by $n$ ! with $\gamma=0.04 \mathrm{eV}$. The black curve is a simulation of the localized exciton MPD rate.

The results of the MPD rates for the first four lowest energy free excitons are shown in Fig. 2a. When the exciton energy matches an integer number of optical phonon quanta, the MPD rate shows a strong resonance, see Fig. 2 2 . The Su-Schreiffer-Heeger type coupling does not break the symmetry of the A and B carbon atom sub-lattices in the CNTs and, therefore, does not allow coupling among even (dark) and odd (bright) parity excitons ${ }^{2}$. The bond-bending term ${ }^{30}$ has a much smaller phonon coupling, but it facilitates the decay of the odd parity states. The bright exciton $(L=0)$ can decay only by emitting phonons whose momenta sum up to zero. This is achieved by a combination of even numbers of zone-boundary optical phonons (D-modes of about 0.18 $\mathrm{eV}$ ) with opposite momenta and arbitrary numbers of Raman active G-phonons with zero momentum.

Considering the limit of a localized single exciton state coupled to a single phonon mode, the MPD decay reduces to the classical Franck-Condon problem, which is exactly solvable in this case $\underline{10}$ :

$$
\frac{1}{\tau_{M P D}}=\frac{2 \pi}{\hbar} \frac{S^{n-1}}{\pi(n-1) !} e^{-S} \frac{n^{2} \gamma S_{I} \hbar^{2} \omega^{2}}{(E-n \hbar \omega)^{2}+n^{2} \gamma^{2}},
$$

where $E$ is exciton energy, $S_{I}$ and $S$ are the interband and intraband Huang-Rhys factors ${ }^{31}$, and $n$ is the number of emitted phonons. The phonon density of states and phonon lifetime are modeled by the broadening parameter $\gamma$. The interband exciton-phonon coupling changes the number of vibrational quanta by one, while the intraband coupling determines the overlap between vibrationally excited state with $n-1$ phonons and vibrational ground state.

The main difference between the calculated MPD rate and the rate estimated according to Eq. (1D) is the substantially smaller magnitude of the former. In CNTs, in the emission process of a phonon with momentum $q^{\prime}$ by an exciton with momentum $q$, the phase of the exciton-phonon matrix depends on both $q^{\prime}$ and $q$. We find that cross-terms corresponding to the phonon emissions in different orders, leading to the same final state with $n$ phonons, will sum to nearly zero, reducing the total MPD rate by nearly a factor of $n ! 29$.

We have calculated the MPD rate for all chirality semiconducting tubes in the diameter range from $0.8 \mathrm{~nm}$ to $2.0 \mathrm{~nm}$ by averaging the energy dependent decay rates in Fig. 2 a over the exciton distribution. The results are shown in Fig. 2b. We find that only a few large diameter tubes, where three or less optical phonons are needed to annihilate the exciton, have lifetimes in the range of $100 \mathrm{ps}$ to $1 \mathrm{~ns}$, whereas most of the other tubes show a much longer non-radiative lifetime. We can also fit the calculated rates in Fig. 20 to the localized limit expression Eq. (11) reduced by $n$ !. The diameter scaling of the intraband Huang-Rhys factor is similar to that of the optical phonon sideband intensity $27-S \propto 1 / d$, where $d$ is a tube diameter. The interband Huang-Rhys factor for the free e-h pair scales inversely with the length $L$ of the tube $S_{I} \propto\left|M^{4}\right|^{2} / N=A\left|M^{4}\right|^{2} /(d L)$, where we calculate $\left|M^{4}\right|^{2} \approx 0.1 \mathrm{eV}^{2}$ and $N$ is the number of two atoms primitive unit-cells of area $A \approx 5.39 \AA^{2}$ in the super-cell. For a bound exciton, we find that the interband coupling is enhanced by a factor of $L / r_{0}$, where the exciton radius $r_{0}$ depends on the diameter and effective dielectric constant $\varepsilon$ that accounts for screening by both the nanotube itself and the immediate environment $\underline{\underline{13}}$. The choice of $\varepsilon=3.3$ reproduces the experimentally measured exciton binding energy in $\mathrm{CNTs}^{32}$, and we find $r_{0}=1.95 d$ and 
$S_{I} \approx 0.02 / d^{2}$. Therefore, we are left with just one fit parameter $S$ and the best agreement is found for $S=0.14 / d$ in Fig. 2 b.

To model localized exciton MPD decay, we assume that the interband Huang-Rhys factor $S_{I}$ is the same as that calculated for the free excitons. We then calculate the intraband Huang-Rhys factor enhancement ${ }^{31}$ by forcing the four lowest energy excitonic bands to have a flat dispersion, while keeping the strength of the exciton-phonon coupling constant. Depending on the tube diameter, we find an effective $S$ enhancement by 1.4 to 1.8. Using Eq. (11) with $S=0.21 / d$, we obtain the MPD rate of localized excitons shown in Fig. 2b. We find that the increased effective exciton-phonon coupling, due to the reduced exciton dispersion, and the coherent contributions of matrix elements bring the MPD rates for the localized states close to the experimentally measured non-radiative decay values, even for small diameter CNTs.

We now examine electronic non-radiative decay mechanisms. A purely electronic (Auger) decay mechanism ${ }^{33}$ can be viewed as the reverse of an impact excitation process 34 . However, the angular momentum conservation law sets strong restrictions for the Auger decay, such that the $\mathrm{E}_{11}$ bright and dark excitons with zero angular momentum cannot decay through such a process 29 . The higher energy dark exciton with finite angular momentum can decay, but only at a high doping level with the Fermi level lying above the bottom of the second band ${ }^{29}$. We also note that at high excitation densities, excitonexciton annihilation dominates the decay 33,35 . However, even at low excitation densities the experimentally measured decay is fast.

We show here that in the presence of the free carriers in CNTs, an exciton can decay fast by creating a phonon and an intraband electron-hole (e-h) pair, as shown in Fig. 1b. This phonon-assisted indirect exciton ionization (PAIEI) process involves Coulomb and exciton-phonon interactions which are both very strong in CNTs $13,27,28,36,37$. The interband exciton-phonon interaction allows the exciton to cross to the electronic ground state. However, emission of a single phonon is not sufficient to conserve energy. The excess energy is accommodated by producing an e-h pair in the valence band (for a p-doped CNT). The initial excitonic state is described by a two-particle wavefunction of exciton in state $p$ with momentum $q$ : $\left|\Psi_{q}^{p}\right\rangle=\sum_{k} A_{k q}^{p} c_{k+q}^{\dagger} v_{k} \mid G S>$. Here, $\left|A_{k q}^{p}\right|^{2}$ gives the contribution of the final hole state $k$ with energy $\varepsilon_{k}$ to the superposition of states describing the initial exciton state. The PAIEI decay rate is given by Fermi's golden rule as shown by the diagram in Fig. 1 $1 b^{29}$ and is controlled by the overlap in reciprocal space of the excitonic and final hole state wavefunctions $\left|A_{k q}^{p}\right|^{2}$, and by the Fermi level which determines the available final states for scattering.

The computed energy-dependent PAIEI rates are shown in Fig. 3a for the four lowest energy excitons in several nanotubes for a fixed doping level of $\rho=0.21$ $\mathrm{e} / \mathrm{nm}$. Unlike in the MPD case, which shows resonances

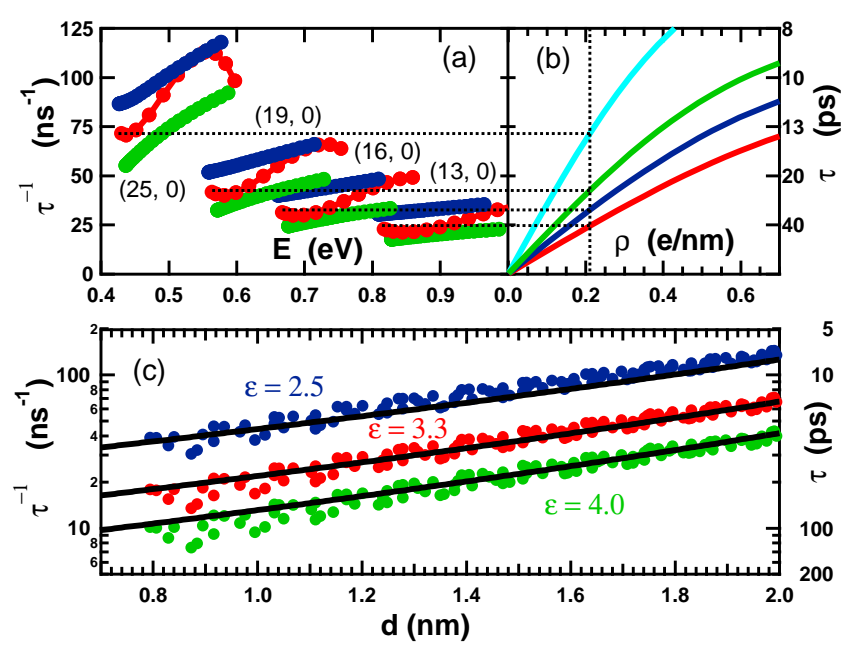

FIG. 3: PAIEI decay rate: (a) as a function of exciton energy for the four lowest energy bands: bright (red), dark (blue), doubly degenerate dark (green); (b) averaged PAIEI decay rate as a function of free carrier density in the same tubes: red - $(13,0)$, blue - $(16,0)$, green - $(19,0)$, and cyan - $(25,0)$ using $\varepsilon=3.3$; (c) averaged PAIEI decay rate in 118 tubes at constant carrier density $\rho=0.21 \mathrm{e} / \mathrm{nm}$, using $\varepsilon=2.5$ (blue circles), $\varepsilon=3.3$ (red circles), $\varepsilon=4.0$ (green circles) along with the simplified expression Eq. (2) (solid lines).

for an integer number of optical phonons, the PAIEI rate shows a much weaker energy dependence. We find that the PAIEI rate at the bottom of the each band in all chirality tubes is largest for the lowest energy dark exciton, while the finite angular momentum, higher energy, dark exciton has the smallest PAIEI rate (Fig. 33). As the doping increases, the PAIEI rate increases proportionally (Fig. 3b).

In the low doping limit, which is the main interest here, the EIPAI rate can be found analytically. The number of final electron states is proportional to the Fermi wavevector $k_{F}$, which defines the free carrier density $\rho$. At zero temperature, $k_{F}=\pi \rho / 4$. This explains the linear dependence of the PAIEI decay rate on $\rho$, which holds up to a $0.4 \mathrm{e} / \mathrm{nm}$ doping level. The number of final hole states is given by the density of states, which we calculate using hyperbolic band dispersion ${ }^{38}$, at the hole energy $\varepsilon_{h} \approx 3 \Delta-\hbar \omega$, where $\Delta \approx 0.42 \mathrm{eV} \mathrm{nm} / d$ is half the bandgap. The tail of the exciton wavefunction in momentum space is the key factor determining the PAIEI rate. In the case of an uncorrelated (free) e$\mathrm{h}$ pair, the two particle wavefunction is a delta function and the PAIEI decay cannot take place. We find that the reciprocal space wavefunction of the lowest energy exciton is best approximated by the following functional form $\left|A_{k, q=0}^{p=1}\right|^{2} \propto\left(1+k^{2} r_{0}^{2}\right)^{-2.629}$. The excitonic radius $r_{0}$ is a fit parameter here and scales with tube diameter $d$ and effective dielectric constant $\varepsilon$ as $r_{0} \approx 0.824 d \varepsilon^{0.72}$. The analytical expression of the PAIEI decay can then 
be written as:

$$
\frac{1}{\tau_{P A I E I}}=\frac{2 \pi}{\hbar} \frac{A S_{1} \hbar^{2} \omega^{2}}{2 \pi C d} \frac{k_{h} r_{0}}{\left(1+k_{h}^{2} r_{0}^{2}\right)^{2.6}} \frac{\rho}{\varepsilon_{h}},
$$

where $k_{h}=\sqrt{\varepsilon_{h}^{2}-\Delta^{2}} /\left(\hbar V_{F}\right), V_{F} \approx 10^{6} \mathrm{~m} / \mathrm{s}$ is the Fermi velocity of graphene, $C \approx 0.65$ is the wavefunction normalization constant, $S_{1}$ is an effective interband HuangRhys factor. Eq. (2) fits remarkably well the PAIEI rate at room temperature and fixed carrier concentration for all chirality semiconducting tubes with diameter range between $0.8 \mathrm{~nm}$ and $2.0 \mathrm{~nm}$ and for a variety of $\varepsilon$ with just one adjustable parameter $S_{1}=2.5$ (Fig. 3. ). This value of $S_{1}$ suggests an electron-phonon coupling $\left|M^{4}\right|^{2}=S_{1} \hbar^{2} \omega^{2}=0.1 \mathrm{eV}^{2}$, which is in good agreement with the calculated value here and measured in 25 . Finally, we also note that the PAIEI decay rate decreases with increasing effective dielectric constant due to the increased exciton radius.
In summary, we have calculated the non-radiative lifetimes of excited semiconducting CNTs via multiphonon decay and by a new, phonon-assisted electronic decay channel, as a function of the CNT diameter. We find that the MPD rate for free excitons is too slow, especially for small diameter CNTs, to account for the experimentally measured decay rates. On the other hand, we predict that the MPD decay rate of localized excitons is increased by a few orders of magnitude, due to a coherent contribution of exciton-phonon matrix elements and a stronger effective exciton-phonon coupling. Thus, localized exciton MPD rates can account for a wide range of available experimental data. Exciton localization is usually associated with charge transfer and doping. In these doped tubes, we find that the decay channel involving phonon-assisted indirect exciton ionization can dominate both the free and localized exciton decay, even at moderately small doping levels.
${ }^{1}$ Ph. Avouris, Z. Chen, V. Perebeinos, Nature Nano 2, 605 (2007).

2 V. Perebeinos, J. Tersoff, and Ph. Avouris, Nano Lett. 5, 2495 (2005).

3 C. D. Spataru et al., Phys. Rev. Lett. 95, 247402 (2005).

4 A. Hagen et al., Appl. Phys. A 78, 1137 (2004).

5 Y.-Z. Ma et al., J. Chem. Phys. 120, 3368 (2004).

${ }^{6}$ G. N. Ostojic et al., Phys. Rev. Lett. 92, 117402 (2004).

7 L. Huang, H. N. Pedrosa, and T. D. Krauss, Phys. Rev. Lett. 93, 017403 (2004).

${ }^{8}$ F. Wang et al., Phys. Rev. Lett. 92, 177401 (2004).

${ }^{9}$ M. Jones et al., Phys. Rev. B 71, 115426 (2005).

${ }^{10} \mathrm{Ph}$. Avouris et al. Physica Status Solidi B 243, 3197 (2006).

11 Carbon Nanotubes, Topics in Applied Physics, edited by A. Jorio, M.S. Dresselhaus, and G. Dresselhaus (SpringerVerlag, New York, 2007).

12 J. Shaver and J. Kono, Laser \& Photonics Reviews 1, 260 (2007)

13 V. Perebeinos, J. Tersoff, and Ph. Avouris, Phys. Rev. Lett. 92, 257402 (2004).

14 H. Zhao, and S. Mazumdar, Phys. Rev. Lett. 93, 157402 (2004).

15 P.Y. Yu and M. Cardona in Fundamentals of Semiconductors (Springer-Verlag, Berlin, 1999).

16 M. Freitag et al., Appl. Phys. Lett. 91, 031101 (2007).

17 L. Cognet et al., Science 316, 1465 (2007).

18 D. A. Tsyboulski et al., Nano Lett. 7, 3080 (2007).

19 A. Hagen et al., Phys. Rev. Lett. 95, 197401 (2005).

20 J. Lefebvre, Y. Homma, and P. Finnie, Phys. Rev. Lett. 90, 217401 (2003)

21 J. Lefebvre et al., Appl. Phys. A 78, 1107 (2004).

22 E. D. Minot et al., Nature 428, 536 (2004).

23 M. S. Fuhrer et al., Nano Lett. 2, 755 (2002).

${ }^{24}$ W. Kim et al., Nano Lett. 3, 193 (2003).

25 J. C. Tsang et al., Nature Nano 2, 725 (2007).

26 R. J. Chen et al., PNAS 100, 4984 (2003).

27 V. Perebeinos, J. Tersoff, and Ph. Avouris, Phys. Rev. Lett 94, $027402(2005)$.
28 M. Lazzeri et al., Phys. Rev. Lett. 95, 236802 (2005).

29 see EPAPS Document No. (appendix attached)

${ }^{30}$ V. Perebeinos, P. B. Allen, and M. Pederson, Phys. Rev. A 72, 012501 (2005).

31 M. Ueta et al., in Excitonic Processes in Solids (SpringerVerlag, Berlin, 2000), Vol. 60.

32 G. Dukovic et al., Nano Lett. 5, 2314 (2005).

33 F. Wang et al., Phys. Rev. B 70, 241403 (R) (2004).

34 V. Perebeinos, and Ph. Avouris, Phys. Rev. B 74, 121410 (R) (2006).

35 Y.-Z. Ma et al., Phys. Rev. Lett. 94, 157402 (2005).

36 T. J. Ando, Phys. Soc. Jpn. 66, 1066 (1997).

37 C. D. Spataru et al., Phys. Rev. Lett 92, 077402 (2004).

38 J. W. Mintmire, B. I. Dunlap, and C. T. White, Phys. Rev. Lett. 68, 631 (1992). 


\section{APPENDIX A: AUGER DECAY}

The Auger decay of the exciton into electron-hole pair involves Coulomb interaction, which is very strong in carbon nanotubes 1,2 . In fact, Auger decay ${ }^{3}$ is a reverse process of the impact excitation, which is much faster in CNTs than in bulk semiconductors ${ }^{4}$. However, for the Auger decay to take place both energy and momenta have to be conserved:

$$
E_{q}^{\Delta L}=e_{k+q}^{l+L}-e_{k}^{l}
$$

where $E$ is the exciton energy and $e$ is the electron and hole energies. The lower index denotes the continuous momentum along the tube axis, and the upper index the discrete angular momentum, which can have values $l=$ $\pm 1, \pm 2, \pm 4$ etc. The angular momentum conservation law sets strong restrictions for the Auger decay. For example, the bright $E_{11}$ exciton with zero angular momentum and $q=0$ can not decay by Auger. Indeed, the right hand side of Eq. (A1) is identically zero for $L=0$ and $q=0$. A similar argument applies for the lowest energy dark exciton with $\Delta L=0$. The higher energy dark excitons ${ }^{5}$ with finite angular momentum $\Delta L=2$ can decay by Auger, but in order to conserve the angular momentum, a hole has to be created in the second band $l_{h}=2$ and an electron in the third band $l_{e}=4$. This requires a high doping level, so that the Fermi level lies above the bottom of the second band. The lowest energy exciton which can decay by Auger at low doping level is $E_{12}$ with $\Delta L=3$, which can be photoexcited with light polarized perpendicular to the tube axis. Indeed, both momentum and energy can be conserved when $E_{12}$ exciton decays into hole in the first band $l_{h}=1$ and electron in the third band $l_{e}=4$ as shown in Fig. 4 .

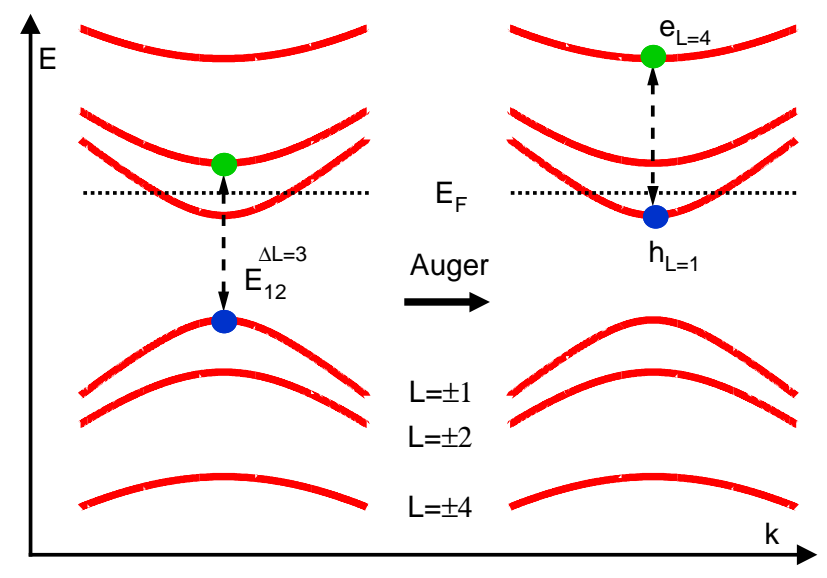

FIG. 4: Schematics of the Auger decay, which can take place at low doping level for a $E_{12}$ exciton with angular momentum $\Delta L=3$. The Fermi level $E_{F}$ is shown by the dashed line. The angular momentum conservation prevents the optically bright exciton $E_{11}(\Delta L=0)$ to decay by this mechanism. The higher energy, dark excitons with $\Delta L=2$ can decay by the Auger mechanism, but only at a high doping level with $E_{F}$ lying above the bottom of the second band.

\section{APPENDIX B: THE MPD RATE CALCULATIONS}

In this section we derive the multiphonon decay (MPD) rate equations using perturbation theory expansion. The transition rate from an initial state $i$ to a final state $f$ is given as:

$$
\begin{aligned}
W_{i f} & =\frac{2 \pi}{\hbar} \delta\left(E_{f}-E_{i}\right)|\langle f|T| i\rangle|^{2} \\
\langle f|T| i\rangle & =\langle f|V| i\rangle+\sum_{k} \frac{\langle f|V| k\rangle\langle k|V| i\rangle}{E_{i}-E_{k}+i \gamma}+\sum_{k k^{\prime}} \frac{\left\langle f|V| k^{\prime}\right\rangle\left\langle k^{\prime}|V| k\right\rangle\langle k|V| i\rangle}{\left(E_{i}-E_{k^{\prime}}+i \gamma\right)\left(E_{i}-E_{k}+i \gamma\right)}+\ldots
\end{aligned}
$$

where $V$ is the exciton-phonon potential and $\gamma$ is the level broadening.

In our case, the initial state exciton two-particle wavefunction $\left|\Psi_{q}^{S}\right\rangle$ with momentum $q$ in state $S$ is given by Eq. (F2) and the final state $\left|\mathrm{GS}, \omega_{q_{1} \mu_{1}}, \omega_{q_{2} \mu_{2}}, \omega_{q_{3} \mu_{3}}, \ldots\right\rangle$ is the electronic ground state $|\mathrm{GS}\rangle$ with a number of created phonons with all possible momenta $q$ and band indexes $\mu$ during the decay process. The number of created phonons in the final state determines the order of the perturbation. 
The total MPD rate of an exciton $(q, S)$ is given by the sum of the first, second etc. orders of perturbation:

$$
W_{q S}=\sum_{i=1}^{n} W_{q S}^{i}
$$

The lowest order non-zero contribution determines the MPD rate. The effective decay rate is averaged over the exciton distribution. In the case when the thermalization is fast compared to the non-radiative lifetime, it is given by:

$$
\bar{W}_{n r}=\frac{1}{Z(T)} \sum_{\nu} W_{\nu} \exp \left(\frac{-E_{\nu}}{k_{B} T}\right)
$$

where index $\nu=(q, \sigma, S)$ includes summation over exciton wavevector $q$, spin index $\sigma$ (labeling singlet and triplet states), and exciton state $S$ (labeling the bound and continuum states for a given $q$ and $\sigma$ ). The partition function is $Z(T)=\sum_{\nu} \exp \left(-E_{\nu} / k_{B} T\right)$, summed over the same range of $\nu$.

Below we give explicit expressions for the first, second, and third order decay rates:

First order (Fermi's Golden rule):

$$
W_{q S}^{1}=\frac{2 \pi}{\hbar} \frac{1}{N} \sum_{\mu} \delta\left(E_{q}^{S}-\hbar \omega_{q \mu}\right)\left|D_{q \mu}^{S}\right|^{2}\left(1+n_{q \mu}\right)
$$

Second order:

$$
\begin{aligned}
W_{q S}^{2}= & \frac{2 \pi}{\hbar} \frac{1}{N} \frac{1}{2 !} \sum_{q_{1} \mu_{1}} \sum_{q_{2} \mu_{2}} \delta\left(E_{q}^{S}-\hbar \omega_{q_{1} \mu_{1}}-\hbar \omega_{q_{2} \mu_{2}}\right) \delta_{q, q_{1}+q_{2}}\left(1+n_{q_{1} \mu_{1}}\right)\left(1+n_{q_{2} \mu_{2}}\right) \\
& \left|\sum_{S_{1}} \frac{B_{q q-q_{1} \mu_{1}}^{S S_{q_{2} \mu_{2}}} D_{S_{1}}^{S_{1}}}{\left(E_{q}^{S}-E_{q-q_{1}}^{S_{1}}-\hbar \omega_{q_{1} \mu_{1}}+i \gamma\right)}+\sum_{S_{2}} \frac{B_{q q-q_{2} \mu_{2}}^{S S_{2}} D_{q_{1} \mu_{1}}^{S_{2}}}{\left(E_{q}^{S}-E_{q-q_{2}}^{S_{2}}-\hbar \omega_{q_{2} \mu_{2}}+i \gamma\right)}\right|^{2}
\end{aligned}
$$

Third order:

$$
\begin{aligned}
W_{q S}^{3}= & \frac{2 \pi}{\hbar} \frac{1}{N} \frac{1}{3 !} \sum_{q_{1} \mu_{1}} \sum_{q_{2} \mu_{2}} \sum_{q_{3} \mu_{3}} \delta\left(E_{q}^{S}-\hbar \omega_{q_{1} \mu_{1}}-\hbar \omega_{q_{2} \mu_{2}}-\hbar \omega_{q_{3} \mu_{3}}\right) \delta_{q, q_{1}+q_{2}+q_{3}}\left(1+n_{q_{1} \mu_{1}}\right)\left(1+n_{q_{2} \mu_{2}}\right)\left(1+n_{q_{3} \mu_{3}}\right) \\
& \left|P_{q_{1} q_{2} q_{3}} \sum_{S_{1} S_{2}} \frac{B_{q q-q_{1} \mu_{1}}^{S S_{1}} B_{q-q_{1} q-q_{1}-q_{2} \mu_{2}}^{S_{1} S_{q_{3} \mu_{3}}} D_{q_{2}}^{S_{2}}}{\left(E_{q}^{S}-E_{q-q_{1}}^{S_{1}}-\hbar \omega_{q_{1} \mu_{1}}+i \gamma\right)\left(E_{q}^{S}-E_{q-q_{1}-q_{2}}^{S_{2}}-\hbar \omega_{q_{1} \mu_{1}}-\hbar \omega_{q_{2} \mu_{2}}+i \gamma\right)}\right|^{2}
\end{aligned}
$$

where $P_{q_{1} q_{2} q_{3}}$ stands for the sum over six permutations of indices $q_{1}, q_{2}$, and $q_{3}$. The interband $B_{q q^{\prime} \mu}^{S S^{\prime}}$ and intraband $D_{q \mu}^{S}$ exciton phonon couplings are given in appendix $\mathrm{F}$.

Note, that in the absence of exciton and phonon dispersions, Eq. (B4)-(B6) reduces to the MPD rate in the localized limit given by Eq. (2) of the main text, except for the missing factor $e^{-S} \approx 1$, which can not be obtained by perturbation theory.

We find that the cross terms in Eq. (B6), corresponding to the different orders of emitted phonons, sum nearly to zero. Therefore, neglecting the interference between these terms is a good approximation to the total MPD rate:

$$
\begin{aligned}
W_{q S}^{n}= & \frac{2 \pi}{\hbar} \sum_{q_{1} \mu_{1}} \sum_{q_{2} \mu_{2}} \ldots \sum_{\mu_{n}} \delta\left(E_{q}^{S}-\hbar \omega_{q-q_{1} \mu_{1}}-\hbar \omega_{q_{1}-q_{2} \mu_{2}}-\ldots-\hbar \omega_{q_{n-1} \mu_{n}}\right) \\
& \left(1+n_{q-q_{1} \mu_{1}}\right)\left(1+n_{q_{1}-q_{2} \mu_{2}}\right) \ldots\left(1+n_{q_{n-1} \mu_{n}}\right)\left|\mathcal{K}_{n}\right|^{2} \\
\mathcal{K}_{n}= & \sum_{S_{1} S_{2} \ldots S_{n-1}} \frac{B_{q q_{1} \mu_{1}}^{S S_{1}}}{\left(E_{q}^{S}-E_{q_{1}}^{S_{1}}-\hbar \omega_{q-q_{1} \mu_{1}}+i \gamma\right)} \frac{B_{q_{1} q_{2} \mu_{2}}^{S_{1} S_{2}}}{\left(E_{q}^{S}-E_{q_{2}}^{S_{2}}-\hbar \omega_{q-q_{1} \mu_{1}}-\hbar \omega_{q_{1}-q_{2} \mu_{2}}+i \gamma\right)} \cdots \\
& \frac{B_{q_{n-2} q_{n-1} \mu_{n-1}}^{S_{n-2} S_{n-1}}}{\left(E_{q}^{S}-E_{q_{n-1}}^{S_{n-1}}-\hbar \omega_{q-q_{1} \mu_{1}}-\hbar \omega_{q_{1}-q_{2} \mu_{2}}-\ldots-\hbar \omega_{q_{n-2}-q_{n-1} \mu_{n-1}}+i \gamma\right)} D_{q_{n-1} \mu_{n}}^{S_{n-1}}
\end{aligned}
$$


The evaluation time of Eq. B7 scales as $N^{n-1}$ which becomes computationally prohibitive for $n \geq 4$. Alternatively, this expression is equivalent to the following set of equations:

$$
\begin{aligned}
F_{1}\left(\varepsilon q S S^{\prime}\right)= & \frac{1}{N} \sum_{\mu} \delta\left(\varepsilon-\hbar \omega_{q \mu}\right)\left(1+n_{q \mu}\right) D_{q \mu}^{S} D_{q \mu}^{* S^{\prime}} \\
F_{N}\left(\varepsilon q S S^{\prime}\right)= & \frac{1}{N} \sum_{q_{1} \mu_{1}} \sum_{S_{1} S_{1}^{\prime}} \int d \varepsilon_{1} \delta\left(\varepsilon-\varepsilon_{1}-\hbar \omega_{q-q_{1} \mu_{1}}\right)\left(1+n_{q-q_{1} \mu_{1}}\right) F_{N-1}\left(\varepsilon_{1} q_{1} S_{1} S_{1}^{\prime}\right) \\
& \frac{B_{q q_{1} \mu_{1}}^{S S_{1}} B_{q q_{1} \mu_{1}}^{* S^{\prime} S_{1}^{\prime}}}{\left(\varepsilon-\hbar \omega_{q-q_{1} \mu_{1}}-E_{q_{1}}^{S_{1}}\right)\left(\varepsilon-\hbar \omega_{q-q_{1} \mu_{1}}-E_{q_{1}}^{S_{1}^{\prime}}\right)} \\
W_{q S}^{N}= & \frac{2 \pi}{\hbar} F_{N}\left(E_{q}^{S}, q, S, S\right)
\end{aligned}
$$

which are used to calculate the MPD rates in Fig. 2 and 3 of the article.

In Fig. 5 we calculate the MPD rate using the exact expression, as in Eq. (B4)-(B6) and neglecting interference terms, as in Eq. (B8). The coherent terms contribute about 1.5 times to the MPD rate for $n=3$ and about 2.5 times for $n=4$, which are significantly lower than in the fully coherent case which give a factor of 6 larger rate for $n=3$ and a factor of 24 for $n=4$.

In the test calculations we replaced the exciton-phonon matrix elements in Eq. (B6) by their absolute values (forcing their phases to be identically zero) and found that the MPD rate for order $n=3$ increases by a factor of 4 , which is much closer to the fully coherent result of $3 !=6$ times larger rate.

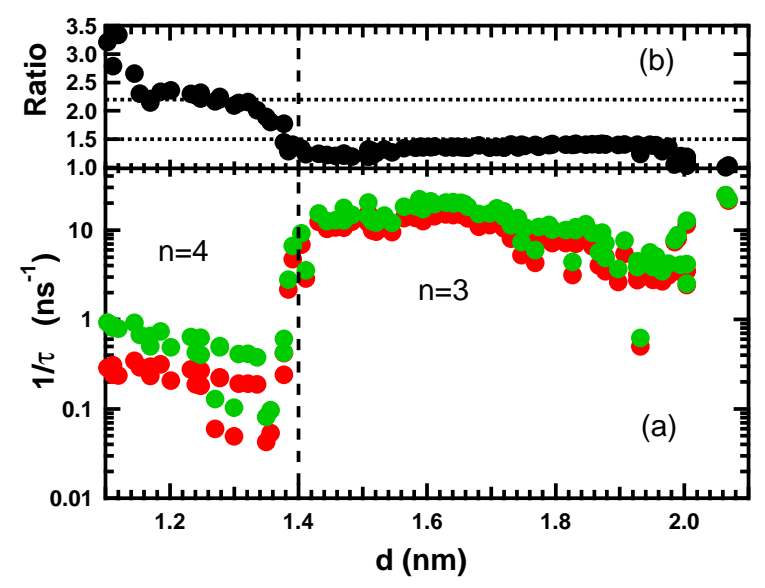

FIG. 5: (a) MPD decay rate of free excitons as a function of tube diameter including coherent contributions (green circles) and neglecting them (red circles). (b) The ratio of the two calculations (black circles) with a two dashed lines corresponding to 1.5 times and 2.2 times rate enhancement due to the coherent contributions for $n=3$ and $n=4$ correspondingly.

\section{APPENDIX C: PAIEI DECAY RATE AND EXCITON WAVEFUNCTION}

In this section, we give the analytical form of the exciton wavefunction in the reciprocal space, which determines the rate of the Phonon-Assisted Indirect Exciton Ionization (PAIEI). Exciton in state $p$ with momentum $q$ and energy $E_{q}^{p}$ can PAIEI decay with the rate given by the Fermi's Golden rule:

$$
\frac{1}{\tau_{P A I E I}^{q p}}=\frac{2 \pi}{N \hbar} \sum_{k_{1} k_{2} \mu}\left|M_{k_{1}+q, k_{2}-k_{1}-q, \mu}^{4}\right|^{2}\left|A_{k_{1} q}^{p}\right|^{2} \delta\left(E_{q}^{p}-\hbar \omega_{q+k_{1}-k_{2}}-\varepsilon_{k_{1}}+\varepsilon_{k_{2}}\right)\left(1+n_{q+k_{1}-k_{2}, \mu}\right) f_{k_{1}}\left(1-f_{k_{2}}\right)
$$

where the Bose-Einstein factor $n_{q}$ gives the phonon population, while the Fermi-Dirac distribution $f_{k}$ defines the probabilities that the hole state with energy and momentum $\left(\varepsilon_{k_{1}}, k_{1}\right)$ and the electron state $\left(\varepsilon_{k_{2}}, k_{2}\right)$ in the valence band are available for scattering. 
The exciton wavefunction $A_{k q}^{p}$ gives the contribution of the final hole state to the superposition of states describing the initial exciton state. We find empirically that it is best fitted by the following equation:

$$
N\left|A_{k q}^{S}\right|^{2}=\frac{\pi^{2}}{2 A C} \frac{d r_{0}}{\left(1+\left(k r_{0}\right)^{2}\right)^{2.6}}
$$

with just one adjustable parameter the exciton radius $r_{0}$. Here, the normalization constant $C=\int_{0}^{\infty} d x /\left(1+x^{2}\right)^{2.6} \approx$ 0.65 requires $\sum_{k}\left|A_{k q}^{S}\right|^{2}=1$, and $A=0.0539 \mathrm{~nm}^{2}$ is the area of the two-atom primitive unit cell, $d$ is the tube diameter, and $N$ is the number of primitive unit cells in the supercell. The results of the fits are excellent, as shown in Fig. 6] for few selected tubes. We find an empirical scaling of the exciton radius with tube diameter and effective dielectric constant $\varepsilon$, that accounts for screening by both the nanotube itself and the immediate environment, to be:

$$
r_{0}=0.824 d \varepsilon^{0.72}
$$

which is similar to the scaling found in ref. $\stackrel{2}{2}$ from the real space wavefunction analysis.

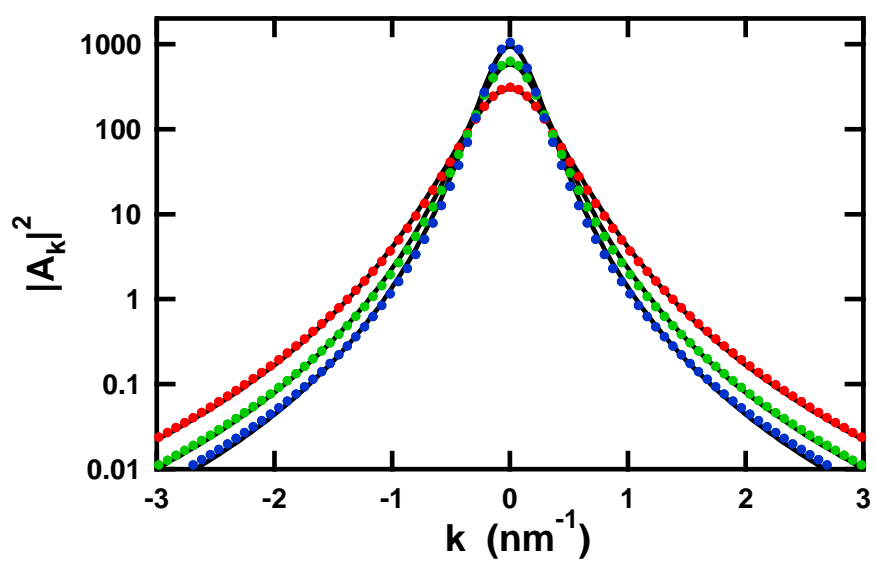

FIG. 6: The lowest energy excitonic wavefunctions in reciprocal space in three tubes $(13,0)$ - red circles, $(19,0)$ - green circles, and $(25,0)$ - red circles calculated for $\varepsilon=3.3$. The black solid curves (shown behind the circles) are best fits to Eq. (C2) with $r_{0}=2.05 \mathrm{~nm}, 2.71 \mathrm{~nm}$, and $3.36 \mathrm{~nm}$, correspondingly.

\section{APPENDIX D: SSH ELECTRON-PHONON MATRIX ELEMENTS}

In this section we give explicit form of the Su-Schreiffer-Heeger (SSH) Hamiltonian 6.7 in reciprocal space. The real space SSH Hamiltonian reads:

$$
\begin{aligned}
\mathcal{H}_{e-p h}^{S S H} & =g \sum_{i \delta} \delta u_{i, i+\delta}\left(C_{i}^{A \dagger} C_{i+\delta}^{B}+C_{i+\delta}^{B \dagger} C_{i}^{A}\right) \\
C_{i}^{A \dagger} & =\frac{1}{\sqrt{N}} \sum_{k} C_{k}^{A \dagger} \exp \left(-i \vec{k} \vec{R}_{i}\right) \\
C_{i+\delta}^{B} & =\frac{1}{\sqrt{N}} \sum_{k} C_{k}^{B} \exp \left(i \vec{k} \vec{R}_{i+\delta}\right) \\
\delta u_{i, i+\delta} & =\vec{n}_{i \delta}\left(\vec{u}_{i+\delta}^{B}-\vec{u}_{i}^{A}\right) \\
\vec{u}_{i}^{A} & =\frac{1}{\sqrt{N}} \sum_{q} \vec{u}_{q}^{A} \exp \left(i \vec{q} \vec{R}_{i}\right) \\
\vec{n}_{i \delta} & =\vec{r}_{i+\delta}^{B}-\vec{r}_{i}^{A},
\end{aligned}
$$

where $R_{i}$ is the position of the unit cell $i ; r_{i}^{A}$ is the position of atom $A$ in unit cell $i$; $N$ is the number of two atom unit cells in the supercell; $g=5.3 \mathrm{eV} / \AA$ is the electron-phonon coupling 8 . 
The SSH Hamiltonian in reciprocal space is:

$$
\begin{aligned}
\mathcal{H}_{e-p h}^{S S H}= & \frac{g}{\sqrt{N}} \sum_{k q \delta} \vec{n}_{0 \delta}\left(\hat{T}_{\delta} \vec{u}_{q}^{B} \exp \left(i \vec{q}\left(\vec{R}_{\delta}-\vec{R}_{0}\right)\right)-\vec{u}_{q}^{A}\right) \\
& \times\left(C_{k+q}^{A \dagger} C_{k}^{B} \exp \left(i \vec{k}\left(\vec{R}_{\delta}-\vec{R}_{0}\right)\right)+C_{k+q}^{B \dagger} C_{k}^{A} \exp \left(-i(\vec{k}+\vec{q})\left(\vec{R}_{\delta}-\vec{R}_{0}\right)\right)\right),
\end{aligned}
$$

where $\hat{T}_{\delta}$ is the rotation operator acting on displacement vector $\vec{u}_{q}^{B}$ in the $i=0$ unit cell and rotating it to $i=\delta$ unit cell. In the tight-binding basis:

$$
\begin{aligned}
C_{k}^{A \dagger} & =\frac{1}{\sqrt{2}}\left(v_{k}^{\dagger}+c_{k}^{\dagger}\right) \\
C_{k}^{B} & =\frac{1}{\sqrt{2}} \frac{\gamma_{k}}{\left|\gamma_{k}\right|}\left(v_{k}-c_{k}\right) \\
\gamma_{k} & =\sum_{\delta} \exp \left(-i \vec{k}\left(\vec{R}_{\delta}-\vec{R}_{0}\right)\right)
\end{aligned}
$$

the electron-phonon interaction has the form:

$$
\mathcal{H}_{\mathrm{el}-\mathrm{ph}}^{\mathrm{SSH}}=\frac{1}{\sqrt{N}} \sum_{k q \mu}\left(M_{k q \mu}^{1} v_{k+q}^{\dagger} v_{k}-M_{k q \mu}^{2} c_{k+q}^{\dagger} c_{k}+M_{k q \mu}^{3} c_{k+q}^{\dagger} v_{k}-M_{k q \mu}^{4} v_{k+q}^{\dagger} c_{k}\right)\left(a_{q \mu}+a_{-q \mu}^{\dagger}\right)
$$

where

$$
\begin{aligned}
& M_{k q \mu}^{1}=M_{k q \mu}^{2}=\frac{g}{2} \sqrt{\frac{\hbar}{2 M \omega_{q}^{\mu}}} \sum_{\delta} \vec{n}_{0 \delta}\left(\hat{T}_{\delta} \vec{B}_{q \mu} \exp \left(i \vec{q}\left(\vec{R}_{\delta}-\vec{R}_{0}\right)\right)-\vec{A}_{q \mu}\right) \\
& \times\left(\frac{\gamma_{k}}{\left|\gamma_{k}\right|} \exp \left(i \vec{k}\left(\vec{R}_{\delta}-\vec{R}_{0}\right)\right)+\frac{\gamma_{k+q}^{*}}{\left|\gamma_{k+q}\right|} \exp \left(-i(\vec{k}+\vec{q})\left(\vec{R}_{\delta}-\vec{R}_{0}\right)\right)\right) \\
& M_{k q \mu}^{3}=M_{k q \mu}^{4}=\frac{g}{2} \sqrt{\frac{\hbar}{2 M \omega_{q}^{\mu}}} \sum_{\delta} \vec{n}_{0 \delta}\left(\hat{T}_{\delta} \vec{B}_{q \mu} \exp \left(i \vec{q}\left(\vec{R}_{\delta}-\vec{R}_{0}\right)\right)-\vec{A}_{q \mu}\right) \\
& \times\left(\frac{\gamma_{k}}{\left|\gamma_{k}\right|} \exp \left(i \vec{k}\left(\vec{R}_{\delta}-\vec{R}_{0}\right)\right)-\frac{\gamma_{k+q}^{*}}{\left|\gamma_{k+q}\right|} \exp \left(-i(\vec{k}+\vec{q})\left(\vec{R}_{\delta}-\vec{R}_{0}\right)\right)\right)
\end{aligned}
$$

The phonon displacements are written in the second quantized form:

$$
\begin{aligned}
& \vec{u}_{q}^{A}=\sum_{\mu} \sqrt{\frac{\hbar}{2 M \omega_{q}^{\mu}}} \vec{A}_{q \mu}\left(a_{q \mu}+a_{-q \mu}^{\dagger}\right) \\
& \vec{u}_{q}^{B}=\sum_{\mu} \sqrt{\frac{\hbar}{2 M \omega_{q}^{\mu}}} \vec{B}_{q \mu}\left(a_{q \mu}+a_{-q \mu}^{\dagger}\right)
\end{aligned}
$$

where $\vec{A}_{q \mu}$ and $\vec{B}_{q \mu}$ are amplitudes of the vibrations with frequency $\omega_{q}^{\mu}$, and $M$ is carbon mass. 


\section{APPENDIX E: BOND-BENDING ELECTRON-PHONON MATRIX ELEMENTS}

In this section we give explicit form of the bond-bending Hamiltonian in reciprocal space. The bond-bending electron-phonon Hamiltonian in real space is:

$$
\begin{aligned}
\mathcal{H}_{e-p h}^{B B}= & g_{b} \sum_{i \delta_{1} \neq \delta_{2}} \Delta \phi_{A}\left(\delta_{1} i \delta_{2}\right) C_{i+\delta_{1}}^{B \dagger} C_{i+\delta_{2}}^{B}+g_{b} \sum_{i \delta_{1} \neq \delta_{2}} \Delta \phi_{B}\left(\delta_{1} i \delta_{2}\right) C_{i+\delta_{1}}^{A \dagger} C_{i+\delta_{2}}^{A} \\
C_{i}^{A \dagger}= & \frac{1}{\sqrt{N}} \sum_{k} C_{k}^{A \dagger} \exp \left(-i \vec{k}_{R_{i}}\right) \\
\Delta \phi_{A}\left(\delta_{1} i \delta_{2}\right)= & \frac{\cos (\phi) \vec{n}_{A i \delta_{1}}-\vec{n}_{A i \delta_{2}}}{r_{A i \delta_{1}} \sin (\phi)} \vec{u}_{i+\delta_{1}}^{B}+\frac{\cos (\phi) \vec{n}_{A i \delta_{2}}-\vec{n}_{A i \delta_{1}}}{r_{A i \delta_{2}} \sin (\phi)} \vec{u}_{i+\delta_{2}}^{B} \\
& +\frac{\left(r_{A i \delta_{1}}-r_{A i \delta_{2}} \cos (\phi)\right) \vec{n}_{A i \delta_{1}}+\left(r_{A i \delta_{2}}-r_{A i \delta_{1}} \cos (\phi)\right) \vec{n}_{A i \delta_{2}} \vec{u}_{i}^{A}}{r_{A i \delta_{1}} r_{A i \delta_{2}} \sin (\phi)} \\
\vec{u}_{i}^{A}= & \frac{1}{\sqrt{N}} \sum_{q} \vec{u}_{q}^{A} \exp \left(i \vec{q} \vec{R}_{i}\right) \\
\vec{n}_{A i \delta}= & \frac{\vec{r}_{A i \delta}}{\vec{r}_{A i \delta} \mid} \\
\vec{r}_{A i \delta}= & \vec{r}_{i+\delta}^{B}-\vec{r}_{i}^{A}, \\
\cos (\phi)= & \vec{n}_{A i \delta_{1}} \vec{n}_{A i \delta_{2}}
\end{aligned}
$$

where $\Delta \phi$ is the C-C-C bond angle change, $R_{i}$ is the position of the unit cell $i ; r_{i}^{A}$ is the position of atom $A$ in unit cell $i ; N$ is the number of two atom unit cells in the supercell; $g_{b}=0.9 \mathrm{eV} / \mathrm{rad}$ is the electron-phonon coupling 8 .

The bond-bending Hamiltonian in reciprocal space reads:

$$
\begin{aligned}
& \mathcal{H}_{e-p h}^{\text {Bend }}=\frac{g_{b}}{\sqrt{N}} \sum_{k q \delta_{1} \neq \delta_{2}} D_{1}\left(q \delta_{1} \delta_{2}\right) C_{k+q}^{B \dagger} C_{k}^{B} \exp \left(i \vec{k}\left(\vec{R}_{\delta_{2}}-\vec{R}_{0}\right)-i(\vec{k}+\vec{q})\left(\vec{R}_{\delta_{1}}-\vec{R}_{0}\right)\right) \\
& +\frac{g_{b}}{\sqrt{N}} \sum_{k q \delta_{1} \neq \delta_{2}} D_{2}\left(q \delta_{1} \delta_{2}\right) C_{k+q}^{A \dagger} C_{k}^{A} \exp \left(i \vec{k}\left(\vec{R}_{\delta_{2}}-\vec{R}_{0}\right)-i(\vec{k}+\vec{q})\left(\vec{R}_{\delta_{1}}-\vec{R}_{0}\right)\right) \\
& D_{1}\left(q \delta_{1} \delta_{2}\right)=\frac{\cos (\phi) \vec{n}_{A \delta_{1}}-\vec{n}_{A \delta_{2}}}{r_{A \delta_{1}} \sin (\phi)}\left(\hat{T}_{\delta_{1}} \vec{u}_{q}^{B}\right) \exp i \vec{q}\left(\vec{R}_{\delta_{1}}-\vec{R}_{0}\right)+\frac{\cos (\phi) \vec{n}_{A \delta_{2}}-\vec{n}_{A \delta_{1}}}{r_{A \delta_{2}} \sin (\phi)}\left(\hat{T}_{\delta_{2}} \vec{u}_{q}^{B}\right) \exp i \vec{q}\left(\vec{R}_{\delta_{2}}-\vec{R}_{0}\right) \\
& +\frac{\left(r_{A \delta_{1}}-r_{A \delta_{2}} \cos (\phi)\right) \vec{n}_{A \delta_{1}}+\left(r_{A \delta_{2}}-r_{A \delta_{1}} \cos (\phi)\right) \vec{n}_{A \delta_{2}}}{r_{A \delta_{1}} r_{A \delta_{2}} \sin (\phi)} \vec{u}_{q}^{A} \\
& D_{2}\left(q \delta_{1} \delta_{2}\right)=\frac{\cos (\phi) \vec{n}_{B \delta_{1}}-\vec{n}_{B \delta_{2}}}{r_{B \delta_{1}} \sin (\phi)}\left(\hat{T}_{\delta_{1}} \vec{u}_{q}^{A}\right) \exp i \vec{q}\left(\vec{R}_{\delta_{1}}-\vec{R}_{0}\right)+\frac{\cos (\phi) \vec{n}_{B \delta_{2}}-\vec{n}_{B \delta_{1}}}{r_{B \delta_{2}} \sin (\phi)}\left(\hat{T}_{\delta_{2}} \vec{u}_{q}^{A}\right) \exp i \vec{q}\left(\vec{R}_{\delta_{2}}-\vec{R}_{0}\right) \\
& +\frac{\left(r_{B \delta_{1}}-r_{B \delta_{2}} \cos (\phi)\right) \vec{n}_{B \delta_{1}}+\left(r_{B \delta_{2}}-r_{B \delta_{1}} \cos (\phi)\right) \vec{n}_{B \delta_{2}}}{r_{B \delta_{1}} r_{B \delta_{2}} \sin (\phi)} \vec{u}_{q}^{B}
\end{aligned}
$$

where $\hat{T}_{\delta}$ is the rotation operator, which rotates carbon atom in the original unit cell to the carbon atom in the neighboring unit cell $\delta$. In the tight-binding basis Eq. (D3) the bond-bending coupling is:

$$
\mathcal{H}_{\mathrm{el}-\mathrm{ph}}=\frac{1}{\sqrt{N}} \sum_{k q \mu}\left(M_{k q \mu}^{1} v_{k+q}^{\dagger} v_{k}-M_{k q \mu}^{2} c_{k+q}^{\dagger} c_{k}+M_{k q \mu}^{3} c_{k+q}^{\dagger} v_{k}-M_{k q \mu}^{4} v_{k+q}^{\dagger} c_{k}\right)\left(a_{q \mu}+a_{-q \mu}^{\dagger}\right)
$$

where

$$
\begin{aligned}
& M_{k q \mu}^{1}=-M_{k q \mu}^{2}=F_{2}(k q \mu)+F_{1}(k q \mu) \frac{\gamma_{k}}{\left|\gamma_{k}\right|} \frac{\gamma_{k+q}^{*}}{\left|\gamma_{k+q}\right|} \\
& M_{k q \mu}^{3}=-M_{k q \mu}^{4}=F_{2}(k q \mu)-F_{1}(k q \mu) \frac{\gamma_{k}}{\left|\gamma_{k}\right|} \frac{\gamma_{k+q}^{*}}{\left|\gamma_{k+q}\right|}
\end{aligned}
$$


where

$$
\begin{aligned}
& F_{1}(k q \mu)=\frac{g_{b}}{2} \sqrt{\frac{\hbar}{2 M \omega_{q}^{\mu}}} \sum_{\delta_{1} \neq \delta_{2}} \exp \left(i \vec{k}\left(\vec{R}_{\delta_{2}}-\vec{R}_{0}\right)-i(\vec{k}+\vec{q})\left(\vec{R}_{\delta_{1}}-\vec{R}_{0}\right)\right) \\
& \times\left[\frac{\cos (\phi) \vec{n}_{A \delta_{1}}-\vec{n}_{A \delta_{2}}}{r_{A \delta_{1}} \sin (\phi)}\left(\hat{T}_{\delta_{1}} \vec{B}_{q \mu}\right) \exp i \vec{q}\left(\vec{R}_{\delta_{1}}-\vec{R}_{0}\right)\right. \\
& +\frac{\cos (\phi) \vec{n}_{A \delta_{2}}-\vec{n}_{A \delta_{1}}}{r_{A \delta_{2}} \sin (\phi)}\left(\hat{T}_{\delta_{2}} \vec{B}_{q \mu}\right) \exp i \vec{q}\left(\vec{R}_{\delta_{2}}-\vec{R}_{0}\right) \\
& \left.+\frac{\left(r_{A \delta_{1}}-r_{A \delta_{2}} \cos (\phi)\right) \vec{n}_{A \delta_{1}}+\left(r_{A \delta_{2}}-r_{A \delta_{1}} \cos (\phi)\right) \vec{n}_{A \delta_{2}}}{r_{A \delta_{1}} r_{A \delta_{2}} \sin (\phi)} \vec{A}_{q \mu}\right] \\
& F_{2}(k q \mu)=\frac{g_{b}}{2} \sqrt{\frac{\hbar}{2 M \omega_{q}^{\mu}}} \sum_{\delta_{1} \neq \delta_{2}} \exp \left(i \vec{k}\left(\vec{R}_{\delta_{2}}-\vec{R}_{0}\right)-i(\vec{k}+\vec{q})\left(\vec{R}_{\delta_{1}}-\vec{R}_{0}\right)\right) \\
& \times\left[\frac{\cos (\phi) \vec{n}_{B \delta_{1}}-\vec{n}_{B \delta_{2}}}{r_{B \delta_{1}} \sin (\phi)}\left(\hat{T}_{\delta_{1}} \vec{A}_{q \mu}\right) \exp i \vec{q}\left(\vec{R}_{\delta_{1}}-\vec{R}_{0}\right)\right. \\
& +\frac{\cos (\phi) \vec{n}_{B \delta_{2}}-\vec{n}_{B \delta_{1}}}{r_{B \delta_{2}} \sin (\phi)}\left(\hat{T}_{\delta_{2}} \vec{A}_{q \mu}\right) \exp i \vec{q}\left(\vec{R}_{\delta_{2}}-\vec{R}_{0}\right) \\
& \left.+\frac{\left(r_{B \delta_{1}}-r_{B \delta_{2}} \cos (\phi)\right) \vec{n}_{B \delta_{1}}+\left(r_{B \delta_{2}}-r_{B \delta_{1}} \cos (\phi)\right) \vec{n}_{B \delta_{2}}}{r_{B \delta_{1}} r_{B \delta_{2}} \sin (\phi)} \vec{B}_{q \mu}\right]
\end{aligned}
$$

\section{APPENDIX F: EXCITON-PHONON MATRIX ELEMENTS}

In this section we derive the exciton-phonon Hamiltonian starting with the electron-phonon Hamiltonian:

$$
\mathcal{H}_{\mathrm{el}-\mathrm{ph}}=\frac{1}{\sqrt{N}} \sum_{k q \mu}\left(M_{k q \mu}^{1} v_{k+q}^{\dagger} v_{k}-M_{k q \mu}^{2} c_{k+q}^{\dagger} c_{k}+M_{k q \mu}^{3} c_{k+q}^{\dagger} v_{k}-M_{k q \mu}^{4} v_{k+q}^{\dagger} c_{k}\right)\left(a_{q \mu}+a_{-q \mu}^{\dagger}\right),
$$

where $M_{k q \mu}^{i} \propto g$ is momentum dependent electron-phonon coupling (see appendixes D and E); $a_{-q \mu}^{\dagger}$ is a phonon creation operator with wavevector $-q$ and phonon band index $\mu=1 \ldots 6$; and $N$ is the number of primitive unit cells, each containing two carbons; $c_{k+q}^{\dagger}\left(v_{k}\right)$ creation (annihilation) of an electron in the conduction (valence) band acting on the ground state $|\mathrm{GS}\rangle=\prod_{k} v_{k}^{\dagger}|\mathrm{vac}\rangle$. The indices $k$ and $q$ label both the continuous $1 \mathrm{D}$ wavevector along the tube axis and the band index.

To derive the exciton-phonon matrix elements from Eq. (F1) we need to know the exciton wavefunction given by the solution of the Bethe-Salpeter Equation (BSE) for the two-particle exciton wavefunction 1,2 :

$$
\left|\Psi_{q}^{S}\right\rangle=\sum_{k} A_{k q}^{S} c_{k+q}^{\dagger} v_{k}|\mathrm{GS}\rangle .
$$

Here $A_{k q}^{S}$ is the eigenvector of the $S$ 's state of BSE solution. Using the orthogonality relation of the BSE solution $\left(\sum_{S} A_{k q}^{S} A_{k^{\prime} q}^{S *}=\delta_{k k^{\prime}}\right)$, we obtain:

$$
c_{k+q}^{\dagger} v_{k}=\sum_{S} A_{k q}^{S *}\left|\Psi_{q}^{S}\right\rangle .
$$

Using Eq. (F1 F3), we derive the exciton-phonon Hamiltonian:

$$
\begin{aligned}
\mathcal{H}_{\mathrm{el}-\mathrm{ph}}\left|\Psi_{q}^{S}\right\rangle= & \frac{1}{\sqrt{N}} \sum_{S^{\prime} q^{\prime} \mu} B_{q q^{\prime} \mu}^{S S^{\prime}}\left(\sqrt{n_{q-q^{\prime} \mu}+1}\left|n_{q-q^{\prime} \mu}+1\right\rangle+\sqrt{n_{q^{\prime}-q \mu}}\left|n_{q^{\prime}-q \mu}-1\right\rangle\right)\left|\Psi_{q^{\prime}}^{S^{\prime}}\right\rangle \\
& +\frac{1}{\sqrt{N}} \sum_{\mu} D_{q \mu}^{S}\left(\sqrt{n_{q \mu}+1}\left|n_{q \mu}+1\right\rangle+\sqrt{n_{-q \mu}}\left|n_{-q \mu}-1\right\rangle\right)|\mathrm{GS}\rangle \\
B_{q q^{\prime} \mu}^{S S^{\prime}}= & -\sum_{k}\left[M_{k q^{\prime}-q \mu}^{1} A_{k+q^{\prime}-q q^{\prime}}^{S} A_{k q^{\prime}}^{S^{\prime} *}+M_{k+q q^{\prime}-q \mu}^{2} A_{k, q}^{S} A_{k q^{\prime}}^{S^{\prime} *}\right] \\
D_{q \mu}^{S}= & -\sum_{k} M_{k+q,-q \mu}^{4} A_{k q}^{S},
\end{aligned}
$$


where $n_{q \mu}$ is the Bose-Einstein phonon occupation number. Here we neglect the coupling of the one exciton subspace to the subspace with two excitons. Matrix elements $B_{q q^{\prime} \mu}^{S S^{\prime}}$ are the intraband and $D_{q \mu}^{S}$ are the interband exciton-phonon couplings.

${ }^{1}$ Spataru, C. D., Ismail-Beigi, S., Benedict, L. X., and Louie, S. G. Excitonic effects and optical spectra of single-walled carbon nanotubes. Phys. Rev. Lett 92, 077402 (2004).

2 Perebeinos, V., Tersoff, J., and Avouris, P. Scaling of excitons in carbon nanotubes. Phys. Rev. Lett. 92, 257402 (2004).

3 Wang, F., Dukovic, G., Knoesel, E., Brus, L. E., and Heinz, T. F. Observation of rapid Auger recombination in optically excited semiconducting carbon nanotubes. Phys. Rev. B 70, 241403 (2004).

4 Perebeinos, V. and Avouris, P. Impact excitation by hot carriers in carbon nanotubes. Phys. Rev. B 74, 121410 (2006).

${ }^{5}$ Perebeinos, V., Tersoff, J., and Avouris, P. Radiative lifetime of excitons in carbon nanotubes. Nano Lett. 5, 2495-2499 (2005).

${ }_{7}^{6} \mathrm{Su}$, W. P., Schrieffer, J. R., and Heeger, A. J. Solitons in Polyacetylene. Phys. Rev. Lett. 42, 1698-1701 (1979).

7 Su, W. P., Schrieffer, J. R., and Heeger, A. J. Soliton excitations in polyacetylene. Phys. Rev. B 22, 2099 - 2111 (1980).

${ }^{8}$ Perebeinos, V., Allen, P. B., and Pederson, M. Reexamination of the Jahn-Teller instability in $\mathrm{C}_{6} \mathrm{H}_{6}^{+}$and $\mathrm{C}_{6} \mathrm{H}_{6}^{-}$. Physical Review A 72, 012501 (2005). 